\title{
IP Simulation of Micro Gas Flows under 3-D Head Sliders
}

\author{
Jun Li and Ching Shen and Jing Fan \\ Laboratory of High Temperature Gas Dynamics, Institute of Mechanics, Chinese Academy of Science, \\ Beijing 100080, China
}

\begin{abstract}
Gas film lubrication of a three-dimensional flat read-write head slider is calculated using the information preservation (IP) method and the direct simulation Monte Carlo (DSMC) method, respectively. The pressure distributions on the head slider surface at different velocities and flying heights obtained by the two methods are in excellent agreement. IP method is also employed to deal with head slider with three-dimensional complex configuration. The pressure distribution on the head slider surface and the net lifting force obtained by the IP method also agree well with those of DSMC method. Much less (of the order about $10^{2}$ less) computational time (the sum of the time used to reach a steady stage and the time used in sampling process) is needed by the IP method than the DSMC method and such an advantage is more remarkable as the gas velocity decreases.
\end{abstract}

Keywords: read-write head slider, micro flows, DSMC method, IP method. PACS: 47.61.Fg, 47.15.gm, 47.45.Ab.

\section{INTRODUCTION}

In the gap between a read-write head slider and a disk, the desk rotation squeezes air under the slider creating a lifting force that causes it to float above the disk at a certain flying height. The flying height is of the order of one nanometer and varies slightly according to the balance between a pre-load of the suspension and the force and moment generated by the squeezed air. The typical slider length in disc drives is about $1000 \mu \mathrm{m}$ and the width is usually $1 / 10 \sim 1 / 3$ of the length. In order to increase the resolution capacity of the head slider, it is desirable to reduce the flying height. The force and moment generated by the squeezed air are two important parameters in the design of the head slider and need to be calculated precisely. Because the flying height is smaller than the mean free path of the air at STP that is about $65 \mathrm{~nm}$, this problem belongs to the transitional regime. Some successful calculations have been made using the generalized Reynolds equation $[1,2,3]$ and the direct simulation Monte Carlo (DSMC) method $[2,4,5]$, respectively. The DSMC method [6] is an appropriate tool to treat such problems with large $K n$ but the low information to noise ratio for low Mach number flows makes the computational process very time-consuming and only simulation results of schematic head slider of small (not authentic) sizes are available. The information preservation (IP) method [7,8] was proposed to overcome the difficulty of serious statistical noise arising from the thermal velocity. The detailed elucidation of IP method including the treatment of multi-dimensional cases is given in reference [9]. It was successfully applied to many low speed micro flows [10-16] and the IP results compared well with DSMC results, experimental data and kinetic exact theory [17]. Reference [18] gives a comprehensive review of the difficulties in simulating internal flows in MEMS and methods to overcome them, which are adopted in the present work. Many efforts were made to develop more accurate and universal model to update IP temperature [19-21] that made important advancements recently, e.g. Sun and Boyd's analysis based on the Maxwell's transport equation in [20], as well as Masters and Ye's octant flux splitting algorithm in [21].

The IP method has been applied to simulate the 2-D flat slider problem with authentic size [22]. In the present work the IP method is used to solve the flows under 3-D head sliders with the purpose of further extending it to solve the 3-D authentic air bearing lubrication problem. In order to verify the results obtained by the IP method, now a head slider much smaller than a real one is chosen in simulation thus the DSMC results can be attained and used for comparison. Some key issues in implementing IP method are addressed. Then, the results obtained by IP method and comparisons with DSMC method are presented. Finally, some conclusions are given.

CP1084, Rarefied Gas Dynamics: $26^{\text {th }}$ International Symposium, edited by T. Abe (C) 2009 American Institute of Physics 978-0-7354-0615-5/09/\$25.00 


\section{IMPLEMENTATION OF THE IP METHOD}

The main idea and the details in implementing IP method are elucidated in [7,8,9,19]. The IP velocity of a simulated molecule, the IP velocity and density averaged over a cell are denoted as $V_{\text {mol, IP }}, V_{\text {cell,IP}}, \rho_{\text {cell,IP}}$, respectively. It should be emphasized that the variables $V_{\text {cell,IP}}$ and $\rho_{\text {cell,IP }}$ are instantaneous and represent the mean values during a time step. They vary according to the unsteady conservative equations and converge finally to the smooth results. The computational domain is divided into small uniform rectangular parallelepipeds-cells. For some cells incised by the solid boundary, they are divided into two parts and only the part inside the fluid domain is considered whose volume is defined as the effective volume of the cell. In the computational process, the increment of the IP velocity $\Delta V_{I P}$ for each cell during a time step caused by the gradient of pressure is calculated as follows [9]:

$$
\Delta V_{I P, i}=\frac{\Delta t}{\rho_{\text {cell }, I P}} \cdot \frac{\partial p_{c e l l, I P}}{\partial x_{i}}
$$

For cells at the air boundary, the ambient pressure $p_{0}$ is used in Eq. (1). This increment of IP velocity $\Delta V_{I P}$ will be added to the IP velocity of molecules located inside the corresponding cell and also be used to update the IP velocity of that cell.

The increment of IP density of each cell during a time step is calculated as follows [9]:

$$
\Delta \rho_{c e l l, I P}=-\Delta t \cdot \frac{\partial\left(\rho V_{c e l l, I P, j}\right)}{\partial x_{j}}
$$

The global mass conservation is checked by:

$$
\Delta Q_{1}+\Delta Q_{2}+\Delta Q_{3}+\Delta Q_{4}=\sum_{n=1}^{m n c} b s(n) \Delta \rho_{c e l l, I P}^{n} \Omega^{n}
$$

where $m n c$ is the total number of cells in the computational domain, $b s(n)$ equals 1 if the cell $n$ contains effective volume or is wholly inside the fluid domain and equals 0 if not, $\Omega^{n}$ is the effective volume of the cell $n$ and $\Delta Q_{1}, \Delta Q_{2}, \Delta Q_{3}, \Delta Q_{4}$ are the mass fluxes flown into the computational region through the air interfaces (see Fig. 1).

\section{RESULTS AND DISCUSSION}

\section{3-D Flat Head Slider}

Figure 1 shows the configuration of a three-dimensional flat slider. The length of the slider is $4 \mu m$ and its width is $3.3 \mu \mathrm{m}$. The upper plate is stationary and its pitch angle $\alpha$ is $0.01 \mathrm{rad}$. The configuration is the same as in [2]. The lower plate moves in its own plane at a velocity $U$ and the flying height is denoted as $H_{\min }$. Argon is chosen as the gas media in the simulation, the ambient temperature is $T_{0}=273.15 \mathrm{~K}$ and the gas density is $\rho_{0}=1.78 \mathrm{~kg} / \mathrm{m}^{3}$. The time step is $9.43 \times 10^{-11} s$, about half the average time between two collisions. The IP and DSMC simulation processes sample once for each four time step. The computational domain is divided into $200 \times 50 \times 5$ uniform cells because enough number of cells along the length direction is needed to catch the drastic change of pressure near the trailing edge of the slider. Each cell contains about 20 molecules. 


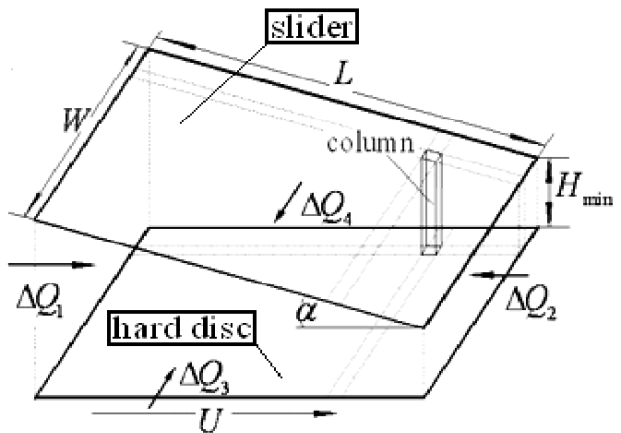

FIGURE 1. Computational domain of a flat head slider.

Figure 2 and 3 show the convergence processes of DSMC and IP calculation, respectively. As shown in figure 2, the simulated molecules accumulate inside the computational domain and their number approaches a steady stage at about $t=0.6 \times 10^{-6} \mathrm{~s}$ (about 6000 time steps). Figure 3 shows that at that moment the mass fluxes from circumstance into the computational domain through four interfaces become steady and the total mass flux $\Delta Q_{1}+\Delta Q_{2}+\Delta Q_{3}+\Delta Q_{4}$ fluctuates around zero. The almost same mass fluxes $\Delta Q_{3}$ and $\Delta Q_{4}$ through two symmetric interfaces at $y=0 \mu m$ and $y=3.3 \mu \mathrm{m}$ are negative which indicate that the molecules flow out of the computational domain. The IP and DSMC sampling processes start at $t=0.6 \times 10^{-6} \mathrm{~s}$. The pressure contours on the slider surface obtained by IP method with 200 sampling time steps and by DSMC method with 200000 sampling time steps are presented in Fig. 4 (left). They agree quite well with each other. To be visual, a three-dimensional distribution is given in Fig. 4 (right) where the normalized IP pressure is taken as z-coordinate.

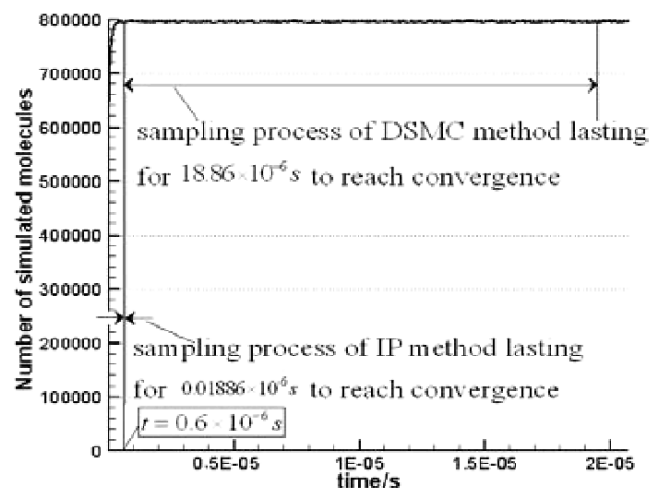

FIGURE 2. The convergence process of DSMC simulation for $H_{\min }=10 \mathrm{~nm}$ and $U=25 \mathrm{~m} / \mathrm{s}$.

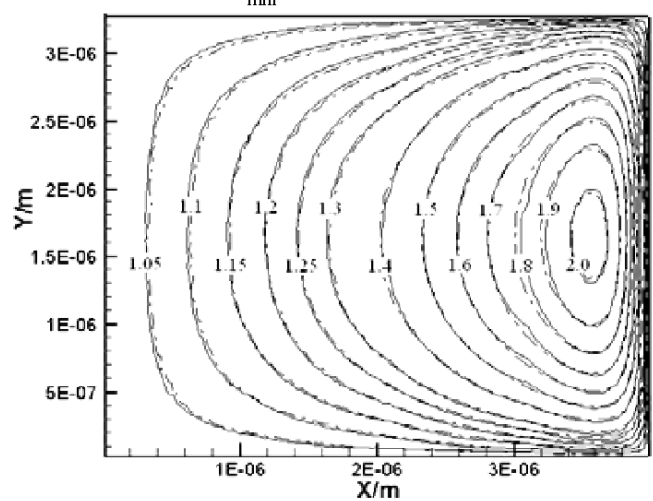

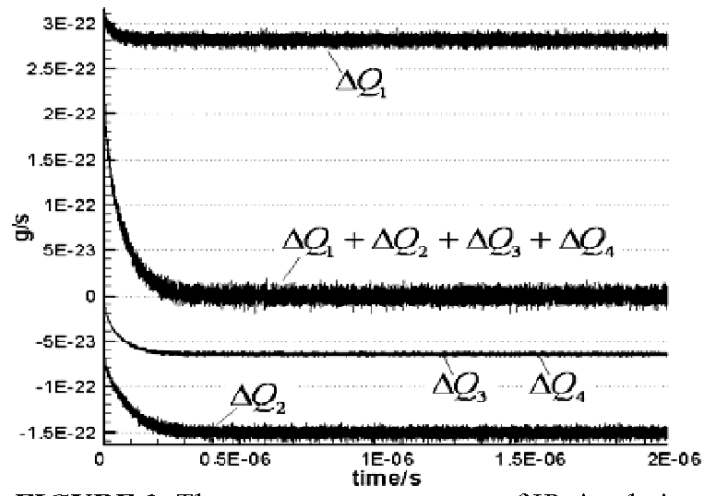

FIGURE 3. The convergence process of IP simulation for $H_{\min }=10 \mathrm{~nm}$ and $U=25 \mathrm{~m} / \mathrm{s}$.

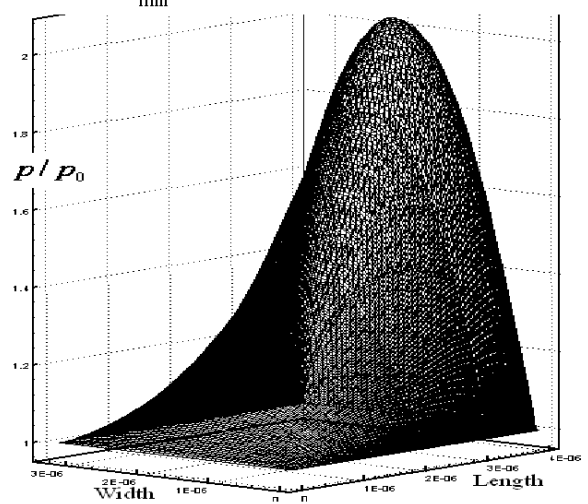

FIGURE 4. The contours of $p / p_{0}$ (left) and the profile of $p / p_{0}$ (right) for $H_{\min }=10 \mathrm{~nm}$ and $U=25 \mathrm{~m} / \mathrm{s}$. Solid line: IP, dashed line: DSMC.

The IP method will become more advantageous as the gas velocity decreases, e.g. the head slider with lower plate 
moving at $U=2 \mathrm{~m} / \mathrm{s}$. The computational domain is divided into $100 \times 50 \times 5$ cells. The IP and DSMC computational processes reach a steady stage at about $t=0.4 \times 10^{-6} \mathrm{~s}$ (about 4000 time steps). Then, the sampling process starts and lasts 600000 time steps for DSMC and 200 time steps for IP respectively. Fig. 5 shows that the DSMC pressure contours with such huge sample size still has obvious scatter in comparison with the IP result that is smooth with much smaller sample size.

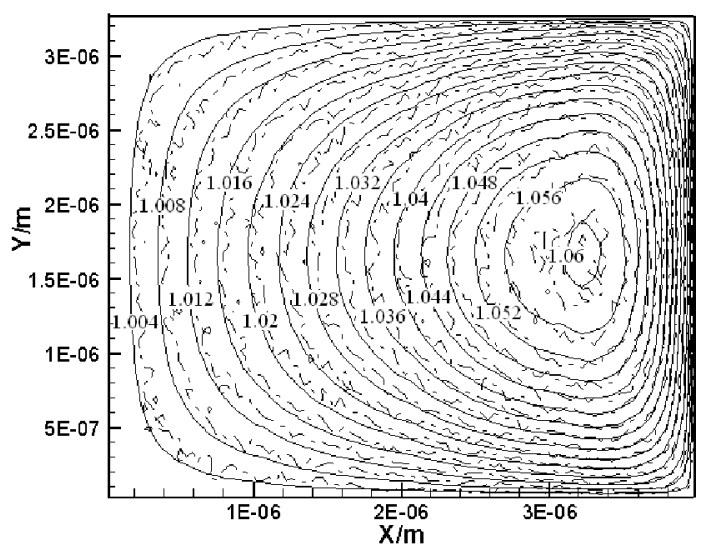

FIGURE 5. The contours of $p / p_{0}$ for $H_{\min }=10 \mathrm{~nm}$ and $U=2 \mathrm{~m} / \mathrm{s}$. Solid line: IP, dashed line: DSMC.

\section{3-D Head Slider with Complex Configuration}

The upper part of Fig. 6 shows a 3-D slider with complex configuration used in our simulation. The geometric size (Fig. 6) is the same order as Fig. 1, so that the results of the DSMC method can be obtained for comparison. The simulated gas is argon, the ambient temperature is $T_{0}=273.15 \mathrm{~K}$ and the density is $\rho_{0}=1.78 \mathrm{~kg} / \mathrm{m}^{3}$. The computational domain is divided into $160 \times 130 \times 26$ uniform cells and each cell contains about 20 molecules. The time step is $7.41 \times 10^{-11} \mathrm{~s}$ about half the mean collision time.

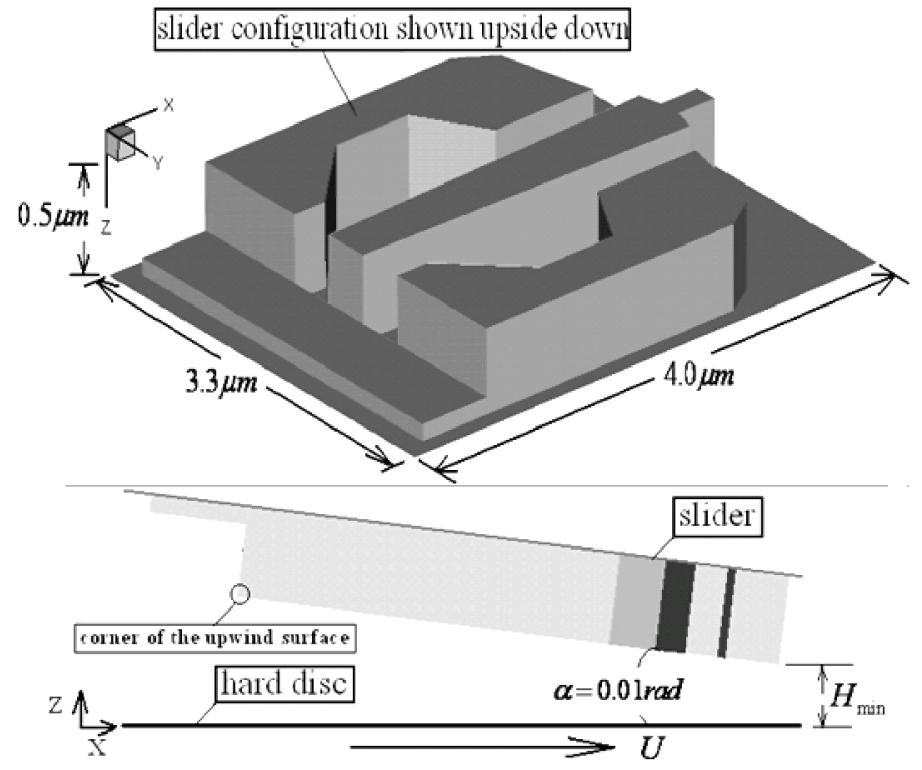

FIGURE 6. Configuration of a head slide used in simulation.

The computational process reaches a steady stage after about 1600 time steps in the case of $H_{\min }=60 \mathrm{~nm}$ and 
$U=10 \mathrm{~m} / \mathrm{s}$. The sampling time steps of DSMC and IP are 160000 and 160 respectively. Figure 7 presents the pressure distribution on the head slider surface obtained by DSMC and IP, respectively. They are generally in good agreement. A small difference takes place in the vicinity of the corner of the upwind surface (Fig. 6) where the pressure change is drastic. The reason is that the corner region contains a singularity point of pressure which will be smoothed unavoidably by any continuous method using finite cells (or nodes).

In this case, the difference between $p$ and $p_{0}\left(p_{0}=101247 P a\right)$ is much smaller than $p_{0}$ itself. This makes the net lifting force contributed by $p-p_{0}$ much smaller than the underlying lifting force due to the ambient pressure $p_{0}$. The net lifting force, however, is most interested in the head slider design. It is a difficult task to calculate precisely the net lifting force. Namely, the difficulty lies in calculating precisely the small difference between two big values. There is a surprising agreement between the net lifting forces given by the IP and DSMC methods that are equal to $8.70 \times 10^{-9} \mathrm{~N}$ and $8.81 \times 10^{-9} \mathrm{~N}$, respectively, while the underlying lifting force is $1.30 \times 10^{-6} \mathrm{~N}$.
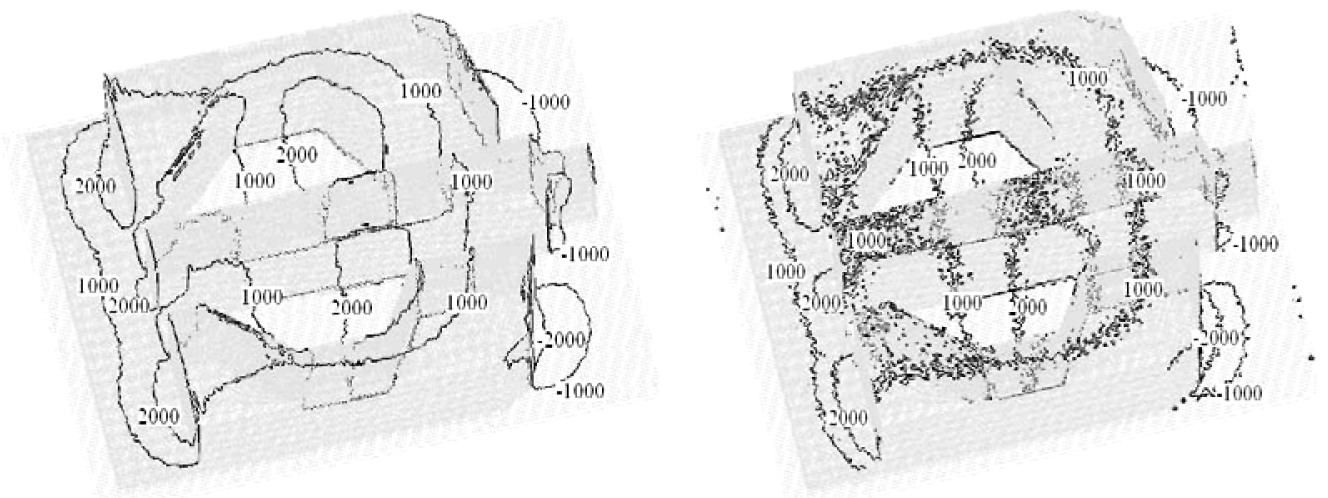

FIGURE 7. The contours of $p-p_{0}(\mathrm{~Pa})$ for $H_{\min }=60 \mathrm{~nm}$ and $U=10 \mathrm{~m} / \mathrm{s}$ by IP method (left) and DSMC method (right).

\section{CONCLUSIONS}

The IP and DSMC method are employed to calculate the pressure distribution over a 3-D flat head slider and a 3-D head slider with complex configuration. In general, the IP and DSMC results are in satisfactory agreement. For the head slider with the complex configuration, the IP and DSMC results are slightly different at certain corner regions that contain a singularity point of pressure. This kind of difference can be reduced through using small cells to locally increase the computational resolution. The total computational time (the sum of the time used to reach a steady stage and the time used in sampling process) required by the IP method to get a smooth result is of the order about $10^{2}$ less than that by the DSMC method. Further, the IP method will be used to analyze the behavior and performance of 3-D head sliders with authentic size.

\section{ACKNOWLEDGEMENTS}

The support of the National Natural Science Foundation of China (Grants 90205024 and 10425211, 621202) is cordially appreciated. Jun Li would like to thank Mr. C. Xie and Dr. Z. J. Jiang for helpful discussions and appreciate the help of Dr. T. S. Liu and Dr. H. C. Wang (from National Chiao Tung University, Hsinchu) for stimulating communications.

\section{REFERENCES}

1. S. Fukui, R. Kaneko, Analysis of ultra-thin gas film lubrication based on linearized Boltzmann equation: first report-derivation of a generalized lubrication equation including thermal creep flow, J. Tribology. 110 (1988) 253-262. 
2. W.D. Huang, D.B. Bogy, Three-dimensional direct simulation Monte Carlo method for slider air bearings, Phys. Fluids 9 (6) (1997) 1764-1769.

3. L. Wu, D.B. Bogy, Numerical simulation of the slider air bearing problem of hard disk dives by two multidimensional upwind residual distribution schemes over unstructured triangular meshes, J Computational Physics 172 (2001) 640-657.

4. Francis J. Alexander, Alejandro L. Garcia, and Berni J. Alder, Direct simulation Monte Carlo for thin-film bearings, Phys. Fluids 6 (12) (1994) 3854-3860.

5. H.C. Wang, T.S. Liu, Airflow velocity effects on air bearing with grooved disk surface in near-field optical disk drives, Phys. Fluids 18 (5) (2006) 057103.

6. G.A. Bird, Molecular Gas Dynamics and the Direct Simulation of Gas Flow, Clarendon Press, 1994.

7. J. Fan, C. Shen (1999) Statistical simulation of low-speed unidirectional flows in transitional regime, Rarefied Gas Dynamics, edited by R Brun, R Campargue, R Gatignol, J C Lengrand, Cepadues, 2: 245-252.

8. J. Fan, C. Shen, Statistical simulation of low-speed rarefied gas flows, J. Computational Physics 167 (2001) 393-412

9. C. Shen, Rarefied Gas Dynamics-Fundamentals, Simulations and Micro Flows, Springer, 2005.

10. C.P. Cai, I.D. Boyd, J. Fan, G.V. Candlir, Direct simulation method for low-speed micro-channel flows, J Thermophysics Heat Transfer, 14 (2000) 368

11. J. Fan, I.D. Boyd, C.P. Cai (2001) Computation of rarefied gas flows around a NACA 0012 airfoil, AIAA J, 39:618.

12. Q.H. Sun, I.D. Boyd, G.V. Candler (2001) Numerical simulation of gas flow over micro-scale airfoils, AIAA paper 2001-3071.

13. H.L. Liu, C. Xie, C. Shen, J. Fan (2001) Flow in membrane filter simulated as microchannel flow with diaphragm, Rarefied Gas Dynamics, edited by T.J. Bartel and M.A. Gallis, 585:524-530.

14. Q.H. Sun, I.D. Boyd, J. Fan (2001) Development of an IP method for subsonic, microscale gas flows, Rarefied Gas Dynamics, edited by T.J. Bartel and M.A. Gallis, 585:547-553

15. J.Z. Jiang, J. Fan, C. Shen (2002) Statistical simulation of micro square cavity flows, Rarefied Gas Dynamics, edited by A.D. Ketsdever and E.P. Muntz, 663: 784-791.

16. C. Shen, J. Fan, C. Xie, Statistical simulation of rarefied gas flows in micrio-channels, J Computational Physics 189 (2003) 512-526.

17. C. Shen, Use of the degenerated Reynolds equation in solving the microchannel flow problem, Physics of Fluids 17 (2005) 046101

18. C. Shen (2004), Information preservation (IP) method in simulation of internal rarefied gas flows in MEMS, Rarefied Gas Dynamics, edited by Mario Capitelli, 762:141-1495.

19. C. Shen, J.Z. Jiang, J. Fan (2001) Information preservation method for the case of temperature variation, Rarefied Gas Dynamics, edited by T.J. Bartel and M. A. Gallis, 585:185-192.

20. Q.H. Sun, I.D. Boyd, Theoretical development of the Information Preservation method for strongly nonequilibrium gas flows, AIAA (2005) 4828

21. N.D. Masters, W.J. Ye, Octant flux splitting information preservation DSMC method for thermally driven flows, J Computational Physics 226 (2007) 2044-2062.

22. J.Z. Jiang, C. Shen, J. Fan (2004) Statistical simulation of thin-film bearings, Rarefied Gas Dynamics, edited by Mario Capitelli, 762: 180-185. 